\title{
FINITE NON-CYCLIC $p$-GROUPS WHOSE NUMBER OF SUBGROUPS IS MINIMAL
}

\author{
STEFANOS AIVAZIDIS ${ }^{\dagger}$ AND THOMAS MÜLLER*
}

\begin{abstract}
A BStRACT. Recent results of Qu and Tărnăuceanu describe explicitly the finite $p$ groups which are not elementary abelian and have the property that the number of their subgroups is maximal among $p$-groups of a given order. We complement these results from the bottom level up by determining completely the non-cyclic finite $p$-groups whose number of subgroups among $p$-groups of a given order is minimal.
\end{abstract}

\section{INTRODUCTION}

Let

$$
M_{p}(1,1,1):=\left\langle a, b, c \mid a^{p}=b^{p}=c^{p}=1,[a, b]=c,[c, a]=[c, b]=1\right\rangle .
$$

Write $\mathfrak{s}_{k}(G)$ for the set of subgroups of order $p^{k}$ of the $p$-group $G$ and $\mathfrak{s}(G)$ for the set of all subgroups of $G$. Recently, $\mathrm{Qu}$ obtained the following result.

Theorem 1.1 ([9, Thm. 1.4]). Let $G$ be a group of order $p^{n}$, where $p$ is an odd prime, and $\widetilde{G}=M_{p}(1,1,1) \times C_{p}^{n-3}$. If $G$ is not elementary abelian, then for all $k$ such that $1 \leqslant k \leqslant n$, we have $\left|\mathfrak{s}_{k}(G)\right| \leqslant\left|\mathfrak{s}_{k}(\widetilde{G})\right|$. In particular, if $2 \leqslant k \leqslant n-2$, then $\left|\mathfrak{s}_{k}(G)\right|<\left|\mathfrak{s}_{k}(\widetilde{G})\right|$.

Thus Qu finds the p-groups whose number of subgroups of possible order is maximal except for elementary abelian $p$-groups when $p>2$. The analogue of Qu's result for $p=2$ was recently obtained by Tărnăuceanu (cf. [10]). We wish to complement Qu's and Tărnăuceanu's results from the bottom level up. In particular, in Theorems A and B we determine completely the structure of those finite non-cyclic $p$-groups whose number of subgroups is minimal.

\section{Finite $p$-GROUPS REALISING The SECOND MINIMAL LEVEL OF SUBGROUP NUMBERS}

We begin with the following preparatory lemma.

Lemma 2.1. Suppose that $G$ is an abelian $p$-group of order $p^{\lambda}$, and that $G$ has $p+1$ subgroups of order $p^{r}$ for all $r$ such that $1 \leqslant r \leqslant \lambda-1$. Then $G \cong C_{p^{\lambda-1}} \times C_{p}$.

Proof. By a well-known result, the number of subgroups of type $\nu=\left(\nu_{1}, \ldots, \nu_{\ell}\right)$ in an abelian $p$-group of type $\mu=\left(\mu_{1}, \ldots, \mu_{m}\right)$ is given by the formula

$$
\prod_{i \geqslant 1} p^{\nu_{i+1}^{\prime}\left(\mu_{i}^{\prime}-\nu_{i}^{\prime}\right)}\left[\begin{array}{l}
\mu_{i}^{\prime}-\nu_{i+1}^{\prime} \\
\nu_{i}^{\prime}-\nu_{i+1}^{\prime}
\end{array}\right]_{p},
$$

2010 Mathematics Subject Classification. 20D15 (20D60). 
where $\mu^{\prime}, \nu^{\prime}$ are the conjugates of the partitions $\mu$ and $\nu$, respectively, and

$$
\left[\begin{array}{l}
n \\
k
\end{array}\right]_{p}=\prod_{i=0}^{k-1} \frac{1-p^{n-i}}{1-p^{i+1}}
$$

is the number of $k$-dimensional subspaces of an $n$-dimensional vector space over the field $\mathbb{Z} / p \mathbb{Z}$; see, for instance, [4, Eqn. (1)]. Since $G$ is non-cyclic of type $\mu=\left(\mu_{1}, \ldots, \mu_{m}\right)$ say, it follows that $m \geqslant 2$ (and $\mu_{2}>0$ ), thus $\mu_{1}^{\prime} \geqslant 2$. Now, let us the count the number of subgroups of type $\nu=(1,0, \ldots, 0)$. Observe that $\nu^{\prime}=(1,0, \ldots, 0)$, so according to formula (2.1) there are

$$
r:=\left[\begin{array}{c}
\mu_{1}^{\prime} \\
1
\end{array}\right]_{p} \cdot \prod_{i \geqslant 2} p^{\nu_{i+1}^{\prime}\left(\mu_{i}^{\prime}-\nu_{i}^{\prime}\right)}\left[\begin{array}{c}
\mu_{i}^{\prime}-\nu_{i+1}^{\prime} \\
\nu_{i}^{\prime}-\nu_{i+1}^{\prime}
\end{array}\right]_{p},
$$

such subgroups. However, $r \leqslant p+1$ since by assumption the number of subgroups of order $p$ in $G$ is $p+1$, and $r \geqslant p+1$, owing to

$$
\left[\begin{array}{c}
\mu_{1}^{\prime} \\
1
\end{array}\right]_{p} \geqslant\left[\begin{array}{l}
2 \\
1
\end{array}\right]_{p}=p+1
$$

Thus $\mu_{1}^{\prime}=2$, which implies that $m=2$ and that $G$ is of type $\mu=\left(\mu_{1}, \mu_{2}\right)$. It follows that

$$
\mu^{\prime}=(\underbrace{2,2, \ldots, 2}_{\mu_{2}}, \underbrace{1,1, \ldots, 1}_{\mu_{1}-\mu_{2}})
$$

Suppose that $\mu_{2} \geqslant 2$. Next, we count subgroups of type $\nu=\left(\mu_{1}-1,1\right)$ in $G$. Note that

$$
\nu^{\prime}=(2, \underbrace{1, \ldots, 1}_{\mu_{1}-1}) \text {. }
$$

Moreover, the second term in the product formula for $\nu=\left(\mu_{1}-1,1\right)$ evaluates to $p$, thus the number of subgroups of type $\nu=\left(\mu_{1}-1,1\right)$ in $G$ is at least $p$. Further, the $\left(\mu_{2}-1\right)$-term in the product formula for $\nu=\left(\mu_{1}, 0\right)$ evaluates to $p$ as well, and thus we deduce that the number of subgroups of type $\nu=\left(\mu_{1}, 0\right)$ in $G$ is, again, at least $p$. Therefore, we get at least $2 p>p+1$ subgroups of order $p^{\mu_{1}}$ in $G$, contrary to our assumption that the number of subgroups of order $p^{\mu_{1}}$ is $p+1$. This contradiction shows that $\mu_{2}=1$ and $\mu_{1}=\lambda-1$, so $G=C_{p^{\lambda-1}} \times C_{p}$ is of the type we asserted.

We shall present our main result as two separate theorems, dealing with the cases $p>2$ and $p=2$ respectively, since the $p=2$ case, although ultimately similar to the $p>2$ case, presents a somewhat erratic behaviour at small values.

Theorem A. Let $p$ be an odd prime, $G$ a p-group of order $p^{\lambda}$. If $G$ is not the cyclic group $C_{p^{\lambda}}$, then $|\mathfrak{s}(G)| \geqslant(p+1)(\lambda-1)+2$, with equality if and only if $G \cong C_{p^{\lambda-1}} \times C_{p}$ or

$$
G \cong M_{p^{\lambda}}:=\left\langle a, b \mid a^{p^{\lambda-1}}=b^{p}=1, a^{b}=a^{1+p^{\lambda-2}}\right\rangle \text {. }
$$

Proof. Given a non-cyclic $p$-group $G, p$ odd, a well-known theorem due to Kulakoff [7, Satz 1] asserts that

$$
\left|\mathfrak{s}_{k}(G)\right| \equiv p+1\left(\bmod p^{2}\right)
$$

for all $k$ such that $1 \leqslant k \leqslant \lambda-1$. Thus, in particular, $\left|\mathfrak{s}_{k}(G)\right| \geqslant p+1$, and therefore

$$
|\mathfrak{s}(G)|=\sum_{k=0}^{\lambda}\left|\mathfrak{s}_{k}(G)\right|=2+\sum_{\substack{k=1 \\ 2}}^{\lambda-1}\left|\mathfrak{s}_{k}(G)\right| \geqslant 2+(\lambda-1)(p+1) .
$$


This proves the first part of our assertion.

Now, we assume (as we may) that $\lambda \geqslant 3$. Applying a result of Lindenberg [8, Folgerung 3.4], we have that if $G=C_{p^{\lambda-1}} \rtimes C_{p}$ (the implied action of $C_{p}$ on $C_{p^{\lambda-1}}$ may well be trivial; we only require that $G$ be a split extension) then $G$ has, apart from the trivial subgroup and the whole group $G, p+1$ subgroups of order $p^{j}, 1 \leqslant j \leqslant \lambda-1$, and thus $\left|\mathfrak{s}\left(C_{p^{\lambda-1}} \rtimes C_{p}\right)\right|=$ $(p+1)(\lambda-1)+2$ for any such split extension.

Our goal now is to establish that if a (necessarily non-cyclic) $p$-group $G$ of order $|G|=p^{\lambda}$ has $p+1$ subgroups of order $p^{j}$, for all $j$ such that $1 \leqslant j \leqslant \lambda-1$, then $G$ is a split extension $C_{p^{\lambda-1}} \rtimes C_{p}$.

If $G$ is abelian, then the claim follows from Lemma 2.1. Now, assume that $G$ is non-abelian. Since $G$ is a $p$-group, $p>2$, this implies that $G$ is not a Dedekind group. Of the $p+1$ subgroups of order $p^{j}$, for each $j$ such that $1 \leqslant j \leqslant \lambda-1$, one is certainly normal, since $p$-groups have normal subgroups of each possible order. The other $p$ are either all normal, or lie in the same conjugacy class. It follows that the non-normal subgroups of $G$ for each possible order are all conjugate, and thus $G$ is a CO-group; cf. top of [2, Sec. 58]. Janko's theorem (see [2, Thm. 58.3]) now yields $G \cong M_{p^{\lambda}}$, where $M_{p^{\lambda}}$ was defined in (2.2). Our proof is complete.

Remark 2.2. We note here that there may, in principle, exist many non-isomorphic semidirect products $C_{p^{\lambda-1}} \rtimes C_{p}$ which are not direct products. However, an early theorem due to Burnside asserts that the only non-abelian p-group, $p$ odd, which has a cyclic maximal subgroup is $M_{p^{\lambda}}$; see [3, Chap. VIII, Sec. 109].

Recall that the generalised quaternion group of order $2^{\lambda}$ is the group defined by the presentation

$$
Q_{2^{\lambda}}:=\left\langle x, y \mid x^{2^{\lambda-1}}=1, y^{2}=x^{2^{\lambda-2}}, x^{y}=x^{-1}\right\rangle .
$$

Next, we address the $p=2$ case.

Theorem B. Let $G$ be a 2-group of order $2^{\lambda}$. If $G$ is not the cyclic group $C_{2^{\lambda}}$ and $\lambda \geqslant 5$, then $|\mathfrak{s}(G)| \geqslant 3 \lambda-1$, with equality if and only if $G \cong C_{2^{\lambda-1}} \times C_{2}$ or $G \cong M_{2^{\lambda}}$, where $M_{2^{\lambda}}$ is as in (2.2) with $p=2$. If $\lambda=3$ and $G$ is not $C_{8}$, then $|\mathfrak{s}(G)| \geqslant 6$ with equality if and only if $G \cong Q_{8}$, while if $\lambda=4$ and $G$ is not $C_{16}$, then $|\mathfrak{s}(G)| \geqslant 11$, with equality if and only if $G \cong Q_{16}$, or $G \cong M_{16}$, or $G \cong C_{8} \times C_{2}$.

Proof. There are 5 groups of order $8=2^{3}$, and 14 groups of order $16=2^{4}$. We use GAP [6] to obtain a full list of the isomorphism classes of groups in each case, and ask GAP for the total number of subgroups of each group in the list. Our claim for $\lambda=3$ and $\lambda=4$ is now a simple matter of inspection.

We may thus assume that $\lambda \geqslant 5$. By Frobenius' generalisation of Sylow's theorem, we have

$$
\left|\mathfrak{s}_{\mu}(G)\right| \equiv 1(\bmod 2),
$$

for all $\mu$ such that $0 \leqslant \mu \leqslant \lambda$.

Moreover, since $G \not C_{2^{\lambda}}$, we have $\left|\mathfrak{s}_{\mu}(G)\right| \neq 1,1 \leqslant \mu \leqslant \lambda-1$ by [1, Prop. 1.3] unless $G \cong Q_{2^{\lambda}}$ and $\mu=1$. It is well-known that $Q_{2^{\lambda}}$ has a unique involution $t$, which generates 
its centre, and affords the quotient $Q_{2^{\lambda}} /\langle t\rangle \cong D_{2^{\lambda-1}}$. Since $\langle t\rangle$ is the unique subgroup of order 2 in $Q_{2^{\lambda}}$, it follows easily that

$$
\left|\mathfrak{s}\left(Q_{2^{\lambda}}\right)\right|=\left|\mathfrak{s}\left(D_{2^{\lambda-1}}\right)\right|+1=\tau\left(2^{\lambda-2}\right)+\sigma\left(2^{\lambda-2}\right)+1=2^{\lambda-1}+\lambda-1,
$$

where $\left|\mathfrak{s}\left(D_{2 m}\right)\right|=\tau(m)+\sigma(m)$ follows from [5, Ex. 1]. Moreover, notice that $\left|\mathfrak{s}\left(Q_{2^{\lambda}}\right)\right| \geqslant$ $3 \lambda-1$ for all $\lambda \geqslant 4$, with equality precisely when $\lambda=4$. Since $\lambda \geqslant 5$, it follows that $G \nsubseteq Q_{2^{\lambda}}$. This shows that $|\mathfrak{s}(G)| \geqslant 3 \lambda-1$ for all $\lambda \geqslant 5$, and by Lemma 2.1 the only abelian group of order $2^{\lambda}$ realising this bound is $C_{2^{\lambda-1}} \times C_{2}$.

It is well known that a 2-group (of order $2^{\lambda}, \lambda \geqslant 5$ ) is Dedekind non-abelian if and only if $G \cong Q_{8} \times C_{2}^{\lambda-3}$. Note here that every group of this type has $G_{0}:=Q_{8} \times\left(C_{2} \times C_{2}\right)$ as a direct factor, and that $\left|\mathfrak{s}_{2}\left(Q_{8} \times C_{2}^{2}\right)\right|=7$. Hence, a non-abelian group $G$ realising the bound $|\mathfrak{s}(G)|=3 \lambda-1$ cannot be Dedekind. By Janko's theorem mentioned previously, the only possible non-abelian group realising the bound $|\mathfrak{s}(G)|=3 \lambda-1$ is $M_{2^{\lambda}}$. But Lindenberg's result applies in the case of $M_{2^{\lambda}}$, since $1+2^{\lambda-2} \not \equiv-1(\bmod 4)$. We conclude that the only groups of order $2^{\lambda}, \lambda \geqslant 5$, which realise the bound $|\mathfrak{s}(G)|=3 \lambda-1$ are $C_{2^{\lambda-1}} \times C_{2}$ and $M_{2^{\lambda}}$. This completes our proof.

\section{REFERENCES}

[1] Y. Berkovich. Groups of prime-power order. Vol. 1, volume 46 of de Gruyter Expositions in Mathematics. Walter de Gruyter GmbH \& Co. KG, Berlin, 2008. With a foreword by Zvonimir Janko.

[2] Y. Berkovich and Z. Janko. Groups of prime-power order. Vol. 2, volume 47 of de Gruyter Expositions in Mathematics. Walter de Gruyter GmbH \& Co. KG, Berlin, 2008.

[3] W. Burnside. On some properties of groups whose orders are powers of primes. Proc. London Math. Soc. (2), 11:225-245, 1913.

[4] L. M. Butler. A unimodality result in the enumeration of subgroups of a finite abelian group. Proc. Amer. Math. Soc., 101(4):771-775, 1987.

[5] W. C. Calhoun. Counting the subgroups of some finite groups. Amer. Math. Monthly, 94(1):54-59, 1987.

[6] The GAP Group, GAP - Groups, Algorithms, and Programming, Version 4.10.1; 2019, (https://www.gap-system.org).

[7] A. Kulakoff. Über die Anzahl der eigentlichen Untergruppen und der Elemente von gegebener Ordnung in p-Gruppen. Math. Ann., 104(1):778-793, 1931.

[8] W. Lindenberg. Über die Struktur zerfallender bizyklischer $p$-Gruppen. J. Reine Angew. Math., 241:118-146, 1970.

[9] H. Qu. Finite non-elementary abelian p-groups whose number of subgroups is maximal. Israel $J$. Math., 195(2):773-781, 2013.

[10] M. Tărnăuceanu. On a conjecture by Haipeng Qu. arXiv e-prints, page arXiv:1811.07478, Nov 2018.

${ }^{*}$ STOCKHOLM, SWEDEN.

E-mail address: stefanosaivazidis@gmail.com

*School of Mathematical Sciences, Queen Mary \& Westfield College, University of London, Mile End Road, London E1 4NS, United Kingdom.

E-mail address: t.w.muller@qmul.ac.uk 